\title{
TWITTER USERS CREDIBLITY EVALUATION BASED ON SOCIAL GRAPH IMPRESSION
}

\author{
Hamda Slimi, Ibrahim Bounhas and Yahya Slimani \\ ENSI, University of Manouba, Tunis, Tunisia \\ LISI, INSAT, University of Carthage, Tunis, Tunisia \\ ISAMM, University of Manouba, Tunis, Tunisia
}

\begin{abstract}
In the era of social media, information became abundant, versatile and easily accessible. Such characteristics enable almost anyone to publish and share their opinion about a variety of subjects. Such freedom comes at a cost since information and source credibility are in jeopardy. Therefore, numerous solutions were proposed to evaluate source credibility. In this paper, we review previous efforts on twitter credibility evaluation. We also propose an approach that relies on the impression of user social network on his tweets to determine his credibility. Furthermore, we incorporate a feature that determines the credibility of the URLs shared by the user through his tweets. The evaluation of our approach has shown it outperforms the baseline in both precision and recall.
\end{abstract}

\section{KEYWORDS}

Information Credibility, Source Credibility, Twitter, Url Score, Social Network

\section{INTRODUCTION}

Twitter, a renowned platform that evolved from a social media to a news outlet in the last decade. It encompasses features that promote an instant and worldwide spread of information. However, twitter lacks gatekeepers to monitor any intentional or non-intentional spread of misinformation. That's why, several research papers have tackled the subject of credibility evaluation of both the content and its authors. Twitter provides to its users a variety of means to interact with a given tweet, namely i) reply to; ii) retweet; and, iii) like (Favorite). The latter allows users to express their opinion and show their impressions on the tweet content. In previous research, these features have been used by to evaluate tweet credibility (Canini et al., 2011) (Castillo et al., 2011).

In (Gupta et al., 2014), authors analyzed user replies to induce an estimate of tweet credibility. They rely on content features, a content verification module and an evaluation of replies polarity to determine the credibility of a newsworthy tweet. Their approach is discussed in detail in section 2. However, the aforementioned features have not been explored to their full extent in the field of user credibility evaluation.

In (Hassan D, 2018), authors focused on detecting political rumor and extreme users. They define an extreme user as one who posts incredible information regularly on a particular topic with some unknown intentions. Also, authors evaluated URL suitability by determining its depth, length in characters, and if the last part contains a dash. Based on these characteristics, they filter out tweets that do not reach certain thresholds. Although, content and user-based features (number of followers, tweeting frequency) were considered in their evaluation of user credibility, authors have neglected features such as the user average number of retweets and favorites which may encompass information about the impression of user social network on his tweets.

To remedy such deficiencies, we propose an approach which relies on the impression of a user social network on his tweets to determine his credibility. Our approach considers features such as the average number of retweets, the average number of replies, the average credibility of shared URLs, the account age, the number of followers and the number of followees to determine the credibility of a user. Furthermore, the accounts that attract our attention belong to ordinary people. These accounts are created by their owners in order to enable them providing and obtaining information through Twitter. In emergency situations people who are present at 
the scene are neither celebrities nor political figures but ordinary citizens. Moreover, we motivate our choice by the fact that accounts belonging to celebrities and new media outlets are not suited for the features we rely on in our approach. In fact, these accounts are deemed credible by the majority of people and require no credibility evaluation.

The remainder of this paper is organized as follows: Section 2 describes existent research on user credibility assessment. In section 3, we detail our proposed approach. Experiments and results are proposed and interpreted in section 4. Finally, section 5 concludes the paper and proposes some ideas for future work.

\section{RELATED WORKS}

A need to detect and remove twitter accounts that operate with malicious intent or political agenda has emerged these last few years (Chang et al., 2016). However, numerous difficulties present themselves such as the intricacies of distinguishing a malicious account based only on its behavior and without human intervention.

Another aspect of the spectrum is spam detection in twitter which often relies on frequency of sharing the same content within a brief period of time to determine if the account is a spammer or not. Finally, user credibility evaluation falls within the range of the aforementioned categories. It relies on content and user features to evaluate credibility. However, credibility is subjective, consequently the nature of the evaluation is dependent on researchers own definition of credibility.

In (Alrubaian et al., 2017), authors presented an approach for twitter user credibility evaluation. It explores features that characterize user expertise and reputation to obtain an evaluation of user credibility. Authors started by measuring user sentiment based on the average sentiment of his tweets. Then, they measured user popularity based on several features such as his social popularity in a certain topic as well as the number of retweets, mentions, and posts in the topic in question. Finally, authors rank users based on their reputation score.

In (SABBEH \& BAATWAH, 2018), authors proposed a novel approach to evaluate credibility of Arabic tweets. Their approach relies on four modules. A content parsing and feature extraction module, a content verification module which checks the veracity of news content in tweet against verified sources. Users' comments polarity evaluation module, and finally a credibility classification module. Authors main contributions are presented in the second and third module. The second module refers to trustworthy Arab news websites and relies on cosine similarity to determine to which extent is the tweet content alike to the trustworthy reference. The third module relies on Arabic sentiment library AraSenTi (Al-Twairesh et al., 2017). It also relies on a set of Arabic and Saudi variant of Arabic words that indicate falsifying or supporting information. An evaluation of the performance of their approach is conducted using a credibility classification module. Authors tested the performance of three classifiers namely; decision tree, SVM, and Naive Bayes. Results show that decision tree outperforms the aforementioned classifiers. Finally, authors compared their results to TweetCred (Gupta et al., 2014) and found that their approach outperformed them. However, TweetCred is not adapted for analyzing newsworthy tweets, instead it is meant for general purpose tweets. Furthermore, in their evaluation of user credibility, authors referred to "Is Verified" feature which as stated by (Edgerly \& Vraga, 2019) little attention is paid to the verification mark when judging credibility.

In (Nourbakhsh et al., 2015), authors developed a framework that infers rumor related cues from tweet and event features. First, authors collected rumor related stories from Emergent.info and @ Snopes which is the twitter feed for the website Snopes.com. The collected rumor stories were filtered and only those that were deemed newsworthy were kept. Then, authors formulated queries based on the previously collected data which were fed to twitter search interface and yielded 421 false stories and their associated 1.47 million tweets. Authors classified topics based on their content into a set of type such as "business and finance, "law and crime", "social issues" etc. They claimed that such a classification would determine to a certain extent which topic are more likely to instigate rumor. Moreover, an emotion-based categorization was applied on topics. Emotions range from alarming or offensive to funny and uplifting. Analysis of the distribution of tweets within the aforementioned categorizations yielded numerous observations like: the predominant emotion is alarming, also stories that had catchy titles also known as clickbait were heavily present in the dataset. Authors also relied on user features, retweet count and retweet rank which is ordered list of tweets based on their retweet count. Finally, authors used Pearsons correlation tests between re-tweet counts and the other factors. Results showed 
a significant correlation between re-tweet counts and follower counts. And that users with high follower counts do not necessarily deliver credible information, since these accounts may also be advertisers or spammers.

Various approaches were proposed in the literature, each one approached the evaluation of twitter user credibility from a different aspect. A thorough analysis of these approaches has shown that none of them analyzed the URL within the user tweets to determine if it impacts his credibility. Also, the impression of his social network on his tweets has not been explored to its full extent. In the upcoming section we will attempt to answer the following research questions. Does URL content and frequency in tweets shared by twitter users within a certain topic impact their topical credibility? Can we derive information about the credibility of the user by analyzing the impression of his social network on his tweets?

\section{PROPOSED APPROACH}

User credibility can be derived from a set of features that describe the user behavior, reputation and social network impression. It was evaluated in previous research papers (Gupta et al., 2014) (Sabbeh \& Baatwah, 2018) (Alrubaian et al., 2017) through the content of his tweets, account age, and tweeting frequency, etc.

In the scope of our study, we focus on determining the propensity of a user to provide valuable, concise and credible content. In order to achieve this, we refer to features provided by Twitter. These features vary from user-based features to tweet-based ones, we combine both of these sets to obtain a representation that incorporate information about user credibility based on his tweets and his interactions.

Twitter enable its users to interact with the tweets they encounter in their feed. First, a user can like a tweet and then marks it as favorited. Also, a tweet can be retweeted it if he deems it as interesting for his followers. Furthermore, a user can reply to a tweet to express his opinion about it. Such interactions encompass information about the tweet credibility as well as its author. The proposed approach relies on information provided by twitter through numerous features to derive an estimation of user credibility.

In fact, we rely on two sets of features. First, basic features which represent data available through the Twitter API such as: number of followers, favorite count and number of tweets. Second, hand crafted features which were derived from the basic features in order to combine the information available within them and convey a more intrinsic elaborate and valuable information about the user behavior.

We perform the following steps. First, basic features are extracted from the JSON document provided by Twitter Search API. Then, features are classified into two categories namely; user features and tweet features. Furthermore, basic features are combined through formulas to attain features that describe user behavior in an eloquent manner. Moreover, features vary in their scale thus a scaling technique is applied to achieve a uniform scale between all features. Finally, a set of classifiers are used to evaluate the performance of the proposed features.

\subsection{Features Extraction}

In our approach, we use two sets of characteristics, namely standard and hand-crafted features. The former, describes the user through his number of followers, number of tweets, number of favorites, account age. The latter are derived from various basic features and are obtained as follows:

- Ratio of likes to number of followers: shows the percentage of user followers that liked (Favorited) his tweets, e.g: a user with 10k followers and an average of 100 likes per tweet, such disparity is a sign of non-valuable content.

- Ratio of retweets to number of followers: shows the percentage of followers that retweeted his tweets to the overall number of his followers. This feature allows us to determine to which extent does the user followers deem his content to be reliable and worthy of sharing.

- Ratio liked to likes: shows the disparity between a user engagement in other tweets and his followers' engagement with his tweets. Such a feature depends on both the tweet and the user. The tweet provides information about the number of likes it has. Whereas, the user provides us with the number of times he favorited other tweets.

- Average sentiments of tweets: for each user we evaluate the average sentiment of his tweets. Using Text Blob library provided in Python we obtain a value from $[-1,1]$ indicating the polarity of a tweet. Then, the average of the obtained values is assigned to the user as his sentiment score. 
- Url feature: previous approaches only consider the presence or absence of a URL in the tweet and consider it as a binary feature (Castillo et al., 2011). The proposed approach evaluates the credibility of a URL within a certain topic by referring to its frequency as shown in formula (1). A popular URL within a topic is ought to be credible since it has been shared by multiple users. Each user has a url score based on the average score of URL of his tweets.

For each $U R L_{i} \in U R L_{T}$ Where: $U R L_{T}=\left\{U R L_{1}, U R L_{1} \ldots U R L_{n_{T}}\right\}$ a set of $n_{T}$ URLs of topic T. We use function (1) to compute URL frequency within a Topic T.

$$
\operatorname{Freq}\left(U R L_{i}\right)=\frac{1}{n_{T}} \sum_{j=1}^{n_{t}}\left[U R L_{i}=U R L_{j}\right]
$$

Each twee $T_{w}$ t contains a set of $\mathrm{URL}_{\mathrm{Tw}}$ of $\mathrm{n}_{\mathrm{Tw}}$ URLs. To compute Tweet URL score we refer to function (2)

$$
\operatorname{Score}\left(U R L_{T w}\right)=\frac{1}{n_{T w}} \sum_{i=1}^{n_{T w}} \operatorname{Freq}\left(U R L_{i}\right)
$$

\subsection{Features Scaling}

The purpose of features scaling is to scale independent variable to a similar range [0-1]. In order to scale the features, we refer to Standard Scaler provided by sklearn in Python. Standards scaler relies on formula (3) to scale features. Where $\mu$ is the mean of the training samples, and $\sigma$ is the standard deviation of the training samples.

Standard Deviation:

$$
z=\frac{x-\mu}{\sigma}
$$

$$
\sigma=\sqrt{\frac{1}{N} \sum_{i=1}^{N}\left(x_{i}-\mu\right)^{2}}
$$

$$
\mu=\frac{1}{N} \sum_{i=1}^{N}\left(x_{i}\right)
$$

\section{EXPERIMENTS AND RESULTS}

In this section, we assess the performance of the proposed features using supervised machine learning approaches. First, a set of six classifiers is selected to classify users based on their credibility. Each user is characterized by a set of features that describe his behavior and the impression of his social graph on his tweets. Second, the performance of each classifier is evaluated on CredBank and Pheme Datasets (cf. section 3.1) via standard metrics. Also, distinct sets of features are used in each iteration to evaluate the contribution of each set (Hand crafted, Basic). Finally, by removing correlated features, an optimal subset is selected based on their contribution to the model performance.

The experiments were conducted using Sklearn library under Python 3.6 (Pedregosa et al., 2011). Furthermore, seven metrics were used based on a 10-fold cross validation to provide an overall evaluation of classifier performance and avoid overfitting.

Scoring and fitting time are intended to show the time for training and predicting the credibility of a user. Although, accuracy is used in previous approaches, it can provide incorrect estimation of classifier performance when the data is unbalanced. For this reason, we refer to Precision, Recall and F1-score. These metrics evaluate the performance of classification of true positive (Credible and predicted Credible), true negative (In-credible and predicted in-credible), false positive (in-Credible and predicted Credible) and false negative (Credible and 
predicted In-credible). For the scope of our study, we aim for the highest recall score since it shows the number of users correctly identified as credible out of the total credible users. The values of AUC_ROC metric range from 0 to 1 , where 0.5 is the score of a random classifier. It allows us to determine to which extent our model is able to distinguish between classes.

\subsection{Dataset Description}

In our approach we use two datasets namely, CredBank (Mitra \& Gilbert, 2015) and Pheme (Zubiaga et al., 2016). The former contains more than 60 million tweets annotated based on their credibility. So, since the dataset size is large and it does not suit the scope of our study, we settle for a subset of tweets and their authors from the overall dataset. As for the latter, we use the entire dataset. Indeed, PHEME was crawled by (Zubiaga et al., 2016) using Twitter streaming API during breaking news events that prompted a propagation of rumors. These events were heavily shared through Twitter at the time of occurrence namely: Sydney Siege, Charlie Hebdo, Germanwings-Crash Ottawa Shooting, and Ferguson Shooting. The tweets that have a high number of retweets were selected. The annotation of the tweets was achieved and verified by a team of journalists. Details about the content of the two datasets is described in Table 1. The database is comprised of four collections describing the users and tweets of each dataset. Each document of the collection contains the features that describe either the user or the tweet.

The aforementioned datasets provide only the credibility annotations of tweets however for our approach we require the users' credibility annotation. That's why, we averaged the credibility annotation of each user tweets. Then, a threshold of 0.6 was empirically selected and any user that had a higher value than the threshold was considered as credible. Table 1 shows the distribution of classes in both datasets.

Table 1. Class distribution for Pheme and Credbank

\begin{tabular}{lll}
\hline Dataset & Number of users & $\begin{array}{l}\text { Credible/Non } \\
\text { Credible Users }\end{array}$ \\
\hline CredBank & 38300 & $26435 / 11865$ \\
Pheme & 02893 & $01869 / 01024$ \\
\hline
\end{tabular}

\subsection{Results of Pheme Dataset}

First, we test the performance of the various combinations of features on Pheme dataset (Zubiaga et al., 2016). The performance of each classifier is evaluated using seven metrics.

\begin{tabular}{lrrrrrrrr} 
& Model & Fitting time & Scoring time & Accuracy & Precision & Recall & F1_score & AUC_ROC \\
\hline 4 & Random Forest & 0.044887 & 0.017243 & 0.685130 & 0.652992 & 0.646560 & 0.681582 & 0.700283 \\
0 & Logistic Regression & 0.034490 & 0.011599 & 0.647453 & 0.607660 & 0.506032 & 0.526070 & 0.566957 \\
2 & Linear Discriminant Analysis & 0.015219 & 0.010554 & 0.646411 & 0.612204 & 0.504894 & 0.524713 & 0.542662 \\
1 & Decision Tree & 0.015621 & 0.009880 & 0.627012 & 0.595029 & 0.596340 & 0.628253 & 0.596340 \\
6 & Bayes & 0.003033 & 0.009698 & 0.624451 & 0.561743 & 0.524490 & 0.545989 & 0.561204 \\
3 & Quadratic Discriminant Analysis & 0.006245 & 0.010240 & 0.623947 & 0.550402 & 0.512363 & 0.539773 & 0.560492 \\
5 & K-Nearest Neighbors & 0.004018 & 0.060467 & 0.589822 & 0.526568 & 0.521711 & 0.573285 & 0.531364
\end{tabular}

Figure 1. Pheme with Basic Features 


\begin{tabular}{rrrrrrrr} 
Model & Fitting time & Scoring time & Accuracy & Precision & Recall & F1_score & AUC_ROC \\
\hline Decision Tree & 0.010277 & 0.008850 & 0.829250 & 0.813579 & 0.813024 & 0.829282 & 0.813024 \\
Random Forest & 0.037784 & 0.014960 & 0.813019 & 0.796057 & 0.794439 & 0.812655 & 0.873626 \\
K-Nearest Neighbors & 0.002818 & 0.044138 & 0.799384 & 0.788127 & 0.759607 & 0.793713 & 0.841150 \\
2uadratic Discriminant Analysis & 0.002685 & 0.009796 & 0.670996 & 0.647120 & 0.641181 & 0.664809 & 0.714361 \\
Logistic Regression & 0.005619 & 0.009152 & 0.670494 & 0.626682 & 0.590959 & 0.643150 & 0.693862 \\
Linear Discriminant Analysis & 0.005878 & 0.009362 & 0.668923 & 0.624128 & 0.589071 & 0.641346 & 0.692195 \\
Bayes & 0.002661 & 0.009274 & 0.653707 & 0.651560 & 0.646662 & 0.651540 & 0.711150
\end{tabular}

Figure 2. Pheme with Hand Crafted Features

\begin{tabular}{rrrrrrrrrr} 
& Model & Fitting time & Scoring time & Accuracy & Precision & Recall & F1_score & AUC_ROC \\
\hline 1 & Decision Tree & 0.019867 & 0.009264 & 0.834560 & 0.820883 & 0.815131 & 0.833688 & 0.815131 \\
4 & Random Forest & 0.048558 & 0.015254 & 0.809895 & 0.793266 & 0.790287 & 0.809427 & 0.868661 \\
& & & & & & & & & \\
5 & K-Nearest Neighbors & 0.003410 & 0.073944 & 0.693081 & 0.659352 & 0.648583 & 0.686693 & 0.708823 \\
0 & Logistic Regression & 0.010593 & 0.009421 & 0.671568 & 0.632394 & 0.599901 & 0.650155 & 0.699530 \\
2 & Linear Discriminant Analysis & 0.011430 & 0.009322 & 0.667906 & 0.627730 & 0.593669 & 0.644762 & 0.693238 \\
6 & Bayes & 0.002938 & 0.009863 & 0.575325 & 0.639525 & 0.633075 & 0.563590 & 0.690250
\end{tabular}

Figure 3. Pheme with combination of both features

Based on Figure 1, we can notice that Random forest reached the best results in all metrics. Basic features were used to obtain these results. As for Figure2, the hand-crafted features were explored and Decision tree was the classifier with the best results. A comparison between Figure 1 and 2 show that hand-crafted features outperform Basic features by $14 \%$ in Accuracy and F1-score. Finally, in Figure 3 Decision tree reaches optimal results with combination of both features sets.

\subsection{Results of CredBank Dataset}

The performance of the proposed features is also tested on CredBank dataset. The goal for testing the performance of the proposed features on distinct datasets is to assure the ability of our model to perform in different contexts and in larger datasets. Such a variance of data source and distribution ascertains the adequacy of the proposed features.

\begin{tabular}{rrrrrrrrr} 
Model & Fitting time & Scoring time & Accuracy & Precision & Recall & F1_score & AUC_ROC \\
\hline Random Forest & 0.498789 & 0.036199 & 0.952722 & 0.955403 & 0.933264 & 0.952132 & 0.970892 \\
K-Nearest Neighbors & 0.040500 & 1.775611 & 0.942831 & 0.939347 & 0.925577 & 0.942378 & 0.959440 \\
Decision Tree & 0.217804 & 0.013769 & 0.930527 & 0.917806 & 0.920036 & 0.930621 & 0.920036 \\
Logistic Regression & 0.094368 & 0.016434 & 0.908965 & 0.892227 & 0.895621 & 0.909162 & 0.942163 \\
Linear Discriminant Analysis & 0.054024 & 0.032253 & 0.894129 & 0.870609 & 0.892248 & 0.895642 & 0.942048 \\
Quadratic Discriminant Analysis & 0.017973 & 0.019504 & 0.546090 & 0.678589 & 0.661049 & 0.536111 & 0.926105 \\
Bayes & 0.007724 & 0.013514 & 0.533825 & 0.674482 & 0.652351 & 0.520787 & 0.924351
\end{tabular}

Figure 4. CredBank with Basic Features 


\begin{tabular}{rrrrrrrr} 
Model & Fitting time & Scoring time & Accuracy & Precision & Recall & F1_score & AUC_ROC \\
\hline Random Forest & 0.276963 & 0.028776 & 0.988843 & 0.987322 & 0.986535 & 0.988839 & 0.998010 \\
Decision Tree & 0.089143 & 0.012778 & 0.987617 & 0.985649 & 0.985328 & 0.987614 & 0.985328 \\
K-Nearest Neighbors & 0.325067 & 0.689006 & 0.982553 & 0.981504 & 0.977511 & 0.982516 & 0.989281 \\
Logistic Regression & 0.072720 & 0.016576 & 0.981208 & 0.978501 & 0.977427 & 0.981199 & 0.977404 \\
Linear Discriminant Analysis & 0.042705 & 0.030828 & 0.979823 & 0.976255 & 0.976497 & 0.979828 & 0.975622 \\
Bayes & 0.006934 & 0.013095 & 0.971792 & 0.964917 & 0.969411 & 0.971862 & 0.972299 \\
Quadratic Discriminant Analysis & 0.009839 & 0.018463 & 0.971792 & 0.964945 & 0.969376 & 0.971861 & 0.972669
\end{tabular}

Figure 5. CredBank with Hand-crafted Features

\begin{tabular}{rrrrrrrr} 
Model & Fitting time & Scoring time & Accuracy & Precision & Recall & F1_score & AUC_ROC \\
\hline Decision Tree & 0.225483 & 0.013043 & 0.990268 & 0.988819 & 0.988398 & 0.990265 & 0.988398 \\
Random Forest & 0.418768 & 0.029700 & 0.988843 & 0.988959 & 0.984893 & 0.988818 & 0.996385 \\
K-Nearest Neighbors & 0.174499 & 3.650786 & 0.986311 & 0.987416 & 0.980517 & 0.986259 & 0.989785 \\
Logistic Regression & 0.143978 & 0.017411 & 0.983067 & 0.981532 & 0.978769 & 0.983041 & 0.990482 \\
Linear Discriminant Analysis & 0.085308 & 0.027024 & 0.981563 & 0.978450 & 0.978423 & 0.981562 & 0.986545 \\
Bayes & 0.008664 & 0.014343 & 0.927796 & 0.906807 & 0.938021 & 0.929175 & 0.981133 \\
Quadratic Discriminant Analysis & 0.022605 & 0.022176 & 0.892901 & 0.870271 & 0.913328 & 0.895919 & 0.979746
\end{tabular}

Figure 6. CredBank with Combination of both Feature Sets

Results shown in Figure 4 to 6 present the same behavior noticed in Pheme dataset with the combination of features outperforming the remaining cases (Basic/Hand-crafted). Also, Random Forest performed the best with basic and hand-crafted features but was outperformed by Decision Tree when both features sets were combined.

Finally, we compare the performance of the proposed approach to (Hassan D, 2018). They used Pheme dataset with a combination of user and content features. Based on Figure 3, we can notice that the Decision tree with a combination of hand-crafted and basic features achieved the best results. Thus, we select it and compare it to Hussain et al. results. Based on Table 2, we can notice that our approach outperforms (Hassan D, 2018) in both Precision and F1-score.

Table 2. Comparison of proposed approach with baseline

\begin{tabular}{|l|l|l|l|}
\hline Approach & Precision & Recall & F1-score \\
\hline Proposed Approach & $\mathbf{0 . 8 2 0}$ & 0.815 & $\mathbf{0 . 8 3 3}$ \\
\hline Hussain et al. & 0.778 & 0.890 & 0.831 \\
\hline
\end{tabular}

The aim of our approach was to answer the research questions established in Section 2. Indeed, Url score has an impact on user credibility, results have shown that the removal of the hand-crafted features drastically reduces the performance of the classifiers. Also, hand crafted features were pertinent enough to impact the results which goes to prove that user follower's impression on his tweets encompasses information about his credibility and the credibility of his tweets.

\section{CONCLUSION}

In this paper, we proposed a novel approach to Twitter user credibility assessment. The proposed approach derives an estimation of user credibility based on his interactions and the impression of his social graph on his tweets. The major contribution for our approach is a set of features that convey information about the impression of user followers on his tweets. The performance of the proposed features was tested on a variety 
of classifiers and datasets. The results have shown that it outperforms the baseline and offers a fast and precise evaluation of user credibility. For future works, we intend to evaluate the frequency by which the user gains new followers and his tweeting regularity throughout his account lifetime. We believe that such characteristics may carry information about the user.

\section{REFERENCES}

Alrubaian, M., Al-Qurishi, M., Al-Rakhami, M., Hassan, M. M., \& Alamri, A. (2017). Reputation-based credibility analysis of Twitter social network users. Concurrency and Computation: Practice and Experience, 29(7), e3873.

Al-Twairesh, N., Al-Khalifa, H., Al-Salman, A., \& Al-Ohali, Y. (2017). Arasenti-tweet: A corpus for arabic sentiment analysis of saudi tweets. Procedia Computer Science, 117, 63-72.

Canini, K. R., Suh, B., \& Pirolli, P. L. (2011, October). Finding credible information sources in social networks based on content and social structure. In 2011 IEEE Third International Conference on Privacy, Security, Risk and Trust and 2011 IEEE Third International Conference on Social Computing (pp. 1-8). IEEE.

Castillo, C., Mendoza, M., \& Poblete, B. (2011, March). Information credibility on twitter. In Proceedings of the 20th international conference on World wide web (pp. 675-684). ACM.

Chang, C., Zhang, Y., Szabo, C., \& Sheng, Q. Z. (2016, December). Extreme user and political rumor detection on twitter. In International Conference on Advanced Data Mining and Applications (pp. 751-763). Springer, Cham.

Edgerly, S., \& Vraga, E. K. (2019). The Blue Check of Credibility: Does Account Verification Matter When Evaluating News on Twitter?. Cyberpsychology, Behavior, and Social Networking, 22(4), 283-287.

Gupta, A., Kumaraguru, P., Castillo, C., \& Meier, P. (2014, November). Tweetcred: Real-time credibility assessment of content on twitter. In International Conference on Social Informatics (pp. 228-243). Springer, Cham.

Hassan, D. (2018, June). A Text Mining Approach for Evaluating Event Credibility on Twitter. In 2018 IEEE 27th International Conference on Enabling Technologies: Infrastructure for Collaborative Enterprises (WETICE) (pp. 171-174). IEEE.

Mitra, T., \& Gilbert, E. (2015, April). Credbank: A large-scale social media corpus with associated credibility annotations. In Ninth International AAAI Conference on Web and Social Media.

Nourbakhsh, A., Liu, X., Shah, S., Fang, R., Ghassemi, M. M., \& Li, Q. (2015, November). Newsworthy rumor events: A case study of twitter. In 2015 IEEE International Conference on Data Mining Workshop (ICDMW) (pp. 27-32). IEEE.

Sabbeh, S. F., \& Baatwah, S. Y. (2018). arabic news credibility on twitter: an enhanced model using hybrid features. journal of theoretical \& applied information technology, 96(8).

Zubiaga, A., Liakata, M., \& Procter, R. (2016). Learning reporting dynamics during breaking news for rumour detection in social media. arXiv preprint arXiv:1610.07363.

Pedregosa, F., Varoquaux, G., Gramfort, A., Michel, V., Thirion, B., Grisel, O., ... \& Vanderplas, J. (2011). Scikit-learn: Machine learning in Python. Journal of machine learning research, 12(Oct), 2825-2830. 